\title{
Combination of known and unknown mechanisms confers high-level resistance to fluoroquinolones in Enterococcus faecium
}

\author{
Yoshihiro Oyamada, ${ }^{1}$ Hideaki Ito, ${ }^{1}$ Kouichi Fujimoto, ${ }^{1}$ Reiko Asada, ${ }^{1}$ \\ Toshiyuki Niga, ${ }^{1}$ Ryoichi Okamoto, ${ }^{2}$ Matsuhisa Inoue ${ }^{2}$ \\ and Jun-ichi Yamagishi ${ }^{1}$ \\ ${ }^{1}$ Pharmacology and Microbiology Research Laboratories, Dainippon Pharmaceutical Co. Ltd, \\ Enoki 33-94, Suita, Osaka 564-0053, Japan \\ ${ }^{2}$ Department of Microbiology, School of Medicine and Environmental Infectious Disease, \\ Graduate School of Medical Sciences, Kitasato University, Kanagawa, Japan
}

Correspondence

Hideaki Ito

hideaki-ito@ds-pharma.co.jp

Received 23 August 2005

Accepted 31 January 2006
In order to elucidate the mechanisms of fluoroquinolone resistance in Enterococcus faecium, spontaneous mutants isolated from Ent. faecium ATCC 19434 by stepwise selection with sparfloxacin (SPX) or norfloxacin (NOR) and 13 clinical isolates of Ent. faecium were characterized by analysing quinolone-resistance-determining regions (QRDRs) of the gyrA, gyrB, parC and parE genes and examining changes in MICs of SPX and NOR in the presence of efflux pump inhibitors. The SPX-selected first-step mutant had a point mutation only in $g y r A$, and the mutants QR7-18 and QR7-39, and clinical isolates that had point mutations in parC, showed NOR resistance. These results indicate that the primary targets of SPX and NOR are DNA gyrase and topoisomerase IV, respectively, and therefore that the primary target of fluoroquinolones in Ent. faecium differs depending on the structure of the compound used. The characterization of the spontaneous mutants and the clinical isolates demonstrates that in addition to the previously reported alterations in GyrA and ParC, an alteration in GyrB, a NorA-like pump, an unknown efflux pump, which excretes both SPX and NOR from bacterial cells, and probably other unknown mechanism(s) all contribute to fluoroquinolone resistance in Ent. faecium.

\section{INTRODUCTION}

Enterococci are important nosocomial pathogens, capable of causing serious life-threatening infections, such as bacteraemia, endocarditis and meningitis (Moellering, 1992). In particular, infections caused by Enterococcus faecium have been on the rise over the past few years (Low et al., 2001; Treitman et al., 2005). Ent. faecium is equipped with a variety of antibiotic-resistance genes, some of which are inherent, while others are acquired. Since many clinical isolates of Ent. faecium are resistant to the majority of antibacterial agents, including vancomycin and fluoroquinolones, therapeutic options for Ent. faecium infections are very limited.

Fluoroquinolone resistance is mediated by alteration of the target enzymes, i.e. DNA gyrase (a heterotetramer in the form $\mathrm{A}_{2} \mathrm{~B}_{2}$ ) and topoisomerase IV (a heterotetramer in the form $\mathrm{C}_{2} \mathrm{E}_{2}$ ), and/or by decreased intracellular accumulation

\footnotetext{
Abbreviations: CCCP, carbonyl cyanide 3-chlorophenylhydrazone; CIP ciprofloxacin; $\mathrm{EtBr}$, ethidium bromide; MDR, multidrug resistance efflux pump; NOR, norfloxacin; ORDR, quinolone-resistance-determining region; SPX, sparfloxacin.
}

of fluoroquinolones (Piddock, 1999). It has already been demonstrated that fluoroquinolone resistance in Enterococcus faecalis and Ent. faecium is associated with mutations in the quinolone-resistance-determining regions (QRDRs) (Yoshida et al., 1990a, 1991) of the gyrA and/or parC genes (Amin et al., 1999; Brisse et al., 1999; Kanematsu et al., 1998; Korten et al., 1994; Tankovic et al., 1996). High-level fluoroquinolone-resistant enterococci have been shown to have amino acid substitutions in GyrA and/or ParC, while no amino acid change has been detected in either GyrA or ParC in the low-level resistant strains (Brisse et al., 1999). Although mutations in other subunit genes in DNA gyrase and topoisomerase IV (i.e. gyrB and parE) have been reported to contribute to the fluoroquinolone resistance of some bacteria, such as Escherichia coli, Staphylococcus aureus and Streptococcus pneumoniae (Piddock, 1999), no report has investigated the association between these mutations and fluoroquinolone resistance in enterococci.

Interestingly, in Gram-negative bacteria such as E. coli, a strain must have a mutation that renders DNA gyrase resistant in order to acquire a subsequent mutation in topoisomerase IV genes, since the primary target of fluoroquinolones in E. coli 
is DNA gyrase (Heisig, 1996). On the other hand, it has been demonstrated that in some Gram-positive organisms, the hierarchy is reversed, and topoisomerase IV is the primary target (Ferrero et al., 1995; Pan et al., 1996). Other studies have shown that the primary target depends on the type of fluoroquinolone (Takenouchi et al., 1995; Morrissey \& George, 1999; Pan et al., 2001).

Efflux is the major cause of decreased intracellular accumulation of fluoroquinolones. For example, in the case of Staph. aureus, the efflux pump involved in fluoroquinolone resistance is NorA (Ng et al., 1994; Yoshida et al., 1990b), while for Strep. pneumoniae, the pump involved is PmrA (Gill et al., 1999). Although a multidrug efflux pump (EmeA), which shows $32 \%$ identity to NorA, has been shown to be associated with Ent. faecalis resistance to fluoroquinolones (Jonas et al., 2001), no information has been provided on the mechanism of Ent. faecium resistance to fluoroquinolones.

In order to elucidate the mechanisms of fluoroquinolone resistance in Ent. faecium, we isolated, in this study, spontaneous fluoroquinolone-resistant mutants from Ent. faecium ATCC 19434 by stepwise selection with sparfloxacin (SPX), a hydrophobic fluoroquinolone, and norfloxacin (NOR), a hydrophilic fluoroquinolone, and characterized the mutants by analysing point mutations in QRDRs of the $g y r A, g y r B$, parC and parE genes, and by comparing MICs of SPX and NOR between the mutants with or without known multidrug efflux pump inhibitors. Furthermore, we characterized 13 clinical isolates of Ent. faecium to examine the extent to which the mechanism(s) of fluoroquinolone resistance found in the mutants isolated in vitro is similar to that in the clinical isolates.

\section{METHODS}

Bacterial strains and culture conditions. Ent. faecium ATCC 19434 was obtained from the American Type Culture Collection (ATCC). The clinical isolates used in this study were collected between 1994 and 1996, identified by an API 20 Strep system (API system) and maintained at Kitasato University. All Ent. faecium strains were grown aerobically at $37^{\circ} \mathrm{C}$ in brain heart infusion broth (Difco Laboratories).

Chemicals and antibacterial agents. SPX and NOR were synthesized at our Chemistry Research Laboratories. Carbonyl cyanide 3-chlorophenylhydrazone (CCCP), ethidium bromide (EtBr), verapamil, lansoprazole and reserpine were purchased from SigmaAldrich Chemical Co. Other reagents were purchased from Nacalai Tesque, unless otherwise indicated.

Determination of MIC. MIC was determined by an agar dilution method with brain heart infusion agar, as recommended by the National Committee for Clinical Laboratory Standards (2003) (now renamed Clinical and Laboratory Standards Institute).

Selection of fluoroquinolone-resistant mutants. Approximately $10^{10}$ c.f.u. of Ent. faecium ATCC 19434 was spread onto brain heart infusion agar containing SPX or NOR at a concentration four times that of the MIC and incubated aerobically at $37^{\circ} \mathrm{C}$ for $48 \mathrm{~h}$. Secondstep mutants were selected in a similar manner from the first-step mutants. All the mutants were derived from selection using a single bacterial culture.

Preparation of total DNA. Ent. faecium total DNA was prepared as described by Hudson \& Curtiss (1990).

PCR amplification and DNA sequence analysis. DNA fragments containing QRDRs of the gyrA, gyrB, parC and parE genes were amplified by PCR with the primers shown in Table 1. PCR was performed with a GeneAmp PCR System 9600 using TAKARA Ex Taq polymerase (Takara Shuzo). The reactions were repeated for 30 cycles $\left(15 \mathrm{~s}\right.$ at $94^{\circ} \mathrm{C}$ for denaturation, $30 \mathrm{~s}$ at $55^{\circ} \mathrm{C}$ for annealing, and $1 \mathrm{~min}$ at $72^{\circ} \mathrm{C}$ for polymerization). PCR-amplified fragments were sequenced by the cycle sequencing method using an ABI Prism Big Dye Terminator version 3.1 Cycle Sequencing kit and then applied to a Genetic analyser 3100 (Applied Biosystems).

Accumulation assays. Accumulation assays were carried out essentially as described by Celesk \& Robillard (1989). The amount of accumulated fluoroquinolones was measured by an agar well diffusion bioassay with E. coli $\mathrm{KP}$ as assay organism (Gonzalez et al., 1985).

Table 1. Primers used for PCR and sequencing

\begin{tabular}{|c|c|c|}
\hline Name & Sequence $\left(5^{\prime}-3^{\prime}\right)$ & Location \\
\hline EFFGA- $1^{*}$ & CGGCGGCACCGTCACCGTCAACAG & bp 139-162 of gyrA \\
\hline EFFGA-2* & GAATTGGGTGTGACACCGGATAAAG & bp $558-579$ of $g y r A$ \\
\hline EFFPC- $1^{\star}$ & TTCCCGTGCATTTCGATCAGTACTTC & bp 185-204 of parC \\
\hline EFFPC-2* & CGTATGACAAAGGATTCCGTAAATC & bp $554-573$ of parC \\
\hline EFFGB- $1 \dagger$ & AGGAAAATTAGCGGACTGCTCT & bp $1242-1263$ of $g y r B$ \\
\hline EFFGB- $2 \dagger$ & GACATCGGCATCAGTCATGA & bp 1511-1530 of $g y r B$ \\
\hline EFFPE-1‡ & CCGTAAAGCAATCAAAGCCAG & bp 1086-1106 of parE \\
\hline EFFPE- $2 \ddagger$ & CGCATCGGTCATGATGATTAC & bp $1450-1470$ of parE \\
\hline
\end{tabular}

${ }^{\star}$ Brisse et al. (1999).

$\dagger$ RefSeq accession number ZP_00286242.

¥RefSeq accession number ZP_00285124. 


\section{RESULTS AND DISCUSSION}

\section{Isolation of fluoroquinolone-resistant mutants of Ent. faecium}

In order to examine the mechanisms of fluoroquinolone resistance in Ent. faecium, a variety of spontaneous fluoroquinolone-resistant mutants were isolated from Ent. faecium ATCC 19434 with SPX, a hydrophobic fluoroquinolone, and NOR, a hydrophilic fluoroquinolone (Fig. 1). When $2 \times$ MIC of SPX and NOR was used, many colonies were obtained, but some were not fluoroquinolone resistant, suggesting that $2 \times$ MIC was too low a concentration to isolate fluoroquinolone-resistant mutants. Therefore, $4 \times$ MIC was used for isolation of the mutants. As shown in Table 2, first-step mutants were obtained at a frequency of $4 \cdot 2 \times 10^{-8}$, regardless of the selection agent. When second-step mutants were selected from the first-step mutants with the same fluoroquinolone that was used for the selection of the first-step mutants, mutants were obtained at a frequency of $1.6 \times 10^{-7}$ with SPX and $1 \cdot 2 \times 10^{-6}$ with NOR. However, the second-step mutants derived from SPX-selected first-step mutants with NOR selection were obtained at a frequency as low as $9 \cdot 0 \times 10^{-9}$, and SPX selection from NOR-selected first-step mutants was not successful. The reason for this difference in secondstep mutant frequency is unclear.

\section{Characterization of first- and second-step mutants}

To characterize the first- and second-step mutants, the association between mutations in the QRDRs of $\operatorname{gy} r A, \operatorname{gyr} B, \operatorname{parC}$ and parE genes and susceptibility to fluoroquinolones was examined. At each selection step, MICs of NOR and SPX for 60 randomly selected isolates were determined, and three mutants selected from the isolates that showed similar MIC patterns were subjected to QRDR analysis. Since the three selected mutants had the same amino acid substitution(s) in QRDR(s) in all cases, one of the mutants was selected as a representative strain (Fig. 1). The characteristics of the
Table 2. Mutant frequency by stepwise selection at fourtimes fluoroquinolone MIC

ND, Not detected.

\begin{tabular}{|lcccc|}
\hline \multicolumn{2}{|c}{ Selection agent } & & \multicolumn{2}{c|}{ Mutant frequency } \\
\cline { 5 - 5 } $\begin{array}{l}\text { First } \\
\text { step }\end{array}$ & $\begin{array}{c}\text { Second } \\
\text { step }\end{array}$ & & $\begin{array}{c}\text { First-step } \\
\text { mutant }\end{array}$ & $\begin{array}{c}\text { Second-step } \\
\text { mutant }\end{array}$ \\
\hline SPX & SPX & & $4 \cdot 2 \times 10^{-8}$ & $1 \cdot 6 \times 10^{-7}$ \\
& NOR & & $9 \cdot 0 \times 10^{-9}$ \\
NOR & SPX & & $4 \cdot 2 \times 10^{-8}$ & ND \\
& NOR & & $1 \cdot 2 \times 10^{-6}$ \\
\hline
\end{tabular}

mutants are summarized in Table 3. SPX-selected first-step mutant QR7 was four times more resistant to SPX than the parent strain, Ent. faecium ATCC 19434, but was as susceptible to NOR as the parent strain. Sequencing analysis of QRDRs revealed that strain QR7 had a Glu-87 (GAG) to Lys (AAG) substitution in GyrA, but no substitution in the QRDRs of GyrB, ParC or ParE. The amino acid change at position 87 of GyrA has already been reported in fluoroquinolone-resistant Ent. faecium and Ent. faecalis (Amin et al., 1999; Kanematsu et al., 1998; Korten et al., 1994). QR19, another first-step mutant, had eightfold decreased susceptibility to NOR, but its susceptibility to SPX was unchanged compared to that of the parent strain. From the results of nucleotide sequencing, QR19 had no mutation in QRDRs of the $g y r A$, gyrB, parC or parE genes. Since hydrophilic quinolones, such as NOR and ciprofloxacin (CIP), are considered to be easily pumped out from bacterial cells by NorA and PmrA, our results suggest that an efflux system like NorA is activated in QR19.

The primary target for fluoroquinolones differs among micro-organisms, depending on the structure of the compound. Which type II topoisomerase is the primary target for the fluoroquinolones in Ent. faecium has not been determined (Amin et al., 1999; Brisse et al., 1999). However, our findings that the SPX-selected first-step mutant

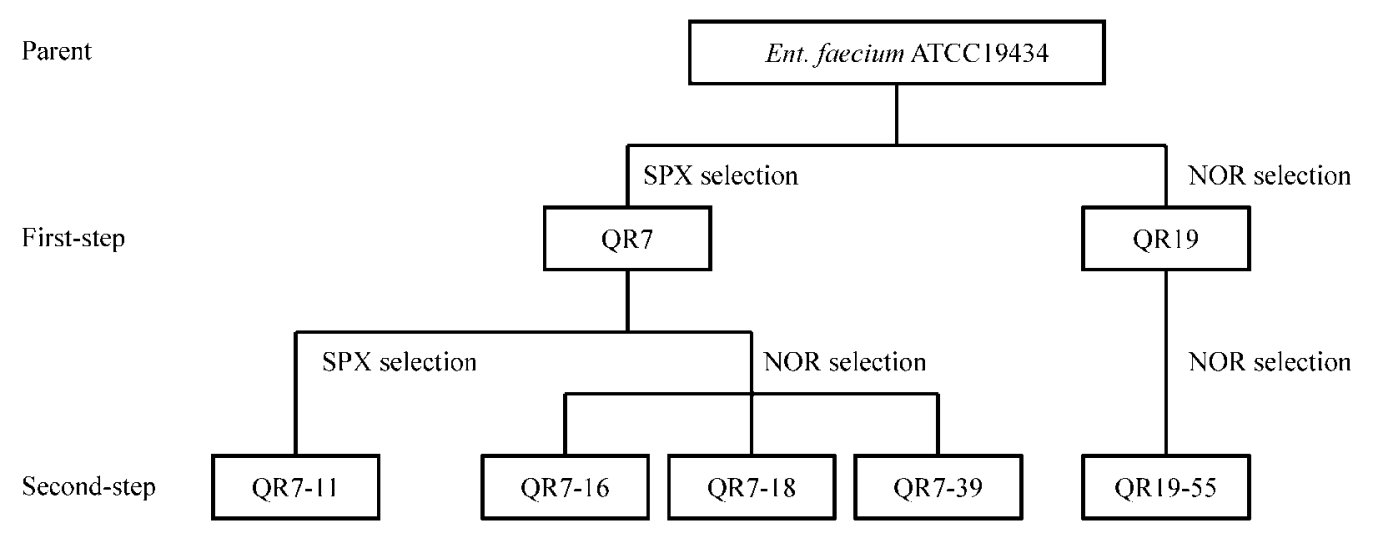

Fig. 1. Schematic representation showing how the first- and second-step mutants were derived from their parent strains. 
Table 3. Topoisomerase subunit changes in first- and second-step fluoroquinolone-resistant Ent. faecium

\begin{tabular}{|c|c|c|c|c|c|c|}
\hline \multirow[t]{2}{*}{ Strain } & \multicolumn{2}{|c|}{$\operatorname{MIC}\left(\mu \mathrm{g} \mathrm{ml}^{-1}\right)$} & \multicolumn{4}{|c|}{ Amino acid substitution in QRDR } \\
\hline & SPX & NOR & GyrA & GyrB & ParC & ParE \\
\hline \multicolumn{7}{|l|}{ First-step mutants } \\
\hline QR7 & 4 & 8 & Glu-87 (GAG) $\rightarrow$ Lys (AAG) & None & None & None \\
\hline QR19 & 1 & 64 & None & None & None & None \\
\hline QR7-11 & 32 & 32 & Glu-87 (GAG) $\rightarrow$ Lys (AAG) & None & None & None \\
\hline QR7-16 & 8 & 32 & Glu-87 (GAG) $\rightarrow$ Lys (AAG) & None & None & None \\
\hline QR7-18 & 128 & $>128$ & Glu-87 (GAG) $\rightarrow$ Lys $($ AAG $)$ & None & Ser-80 (AGC) $\rightarrow$ Ile (ATC) & None \\
\hline QR7-39 & 128 & $>128$ & Glu-87 (GAG) $\rightarrow$ Lys (AAG) & None & Glu-84 (GAA) $\rightarrow$ Lys (AAA) & None \\
\hline \multicolumn{7}{|c|}{ QR19-derived second-step mutant } \\
\hline QR19-55 & 2 & $>128$ & None & None & None & None \\
\hline
\end{tabular}

QR7 had a point mutation only in the gyrA gene, and that the NOR-selected first-step mutant QR19 had no mutation in QRDRs of type II topoisomerase genes, demonstrate that the primary target at least for SPX is DNA gyrase.

The second-step mutants QR7-18 and QR7-39 selected with NOR had 32-fold and $>16$-fold decreased susceptibility to SPX and NOR, respectively, and had amino acid changes in ParC that have previously been found in fluoroquinoloneresistant Ent. faecium and Ent. faecalis, i.e. Ser-80 (AGC) to Ile (ATC) in QR7-18 and Glu-84 (GAA) to Lys (AAA) in QR7-39 (Amin et al., 1999; Brisse et al., 1999; Kanematsu et al., 1998). No QRDR mutation was associated with resistance to NOR, although the gyrA mutant (QR7) possessed the same NOR MIC as the parent strain, suggesting that the primary target for NOR might be topoisomerase IV. As no single parC mutant was obtained, this was not proven conclusively. Taken together with the finding that the primary target for SPX is DNA gyrase, it is suggested that the primary fluoroquinolone target in Ent. faecium as well as in Strep. pneumoniae differs depending on the structure of the compound used (Morrissey \& George, 1999; Pan et al., 2001). The second-step mutants QR7-11 and QR7-16 isolated from the SPX-selected first-step mutant QR7 by SPX and NOR selection, respectively, were two to eight times more resistant to SPX and four times more resistant to NOR than the parent strain, which had a mutation in GyrA. Surprisingly, QR7-11 and QR7-16 had no additional amino acid substitution in QRDRs of GyrA, GyrB, ParC and ParE. The second-step mutant QR19-55 isolated from the NORselected first-step mutant QR19 by NOR selection was two times or more resistant to SPX and NOR than the parent strain. No mutation in QRDRs of gyrA, gyrB, parC and parE genes from QR19-55 was detected. Thus far, the mechanisms of acquisition of fluoroquinolone resistance cannot be explained by QRDR mutations only. Apart from the possibility of mutations in regions other than QRDRs, a multidrug resistance efflux pump (MDR) might be involved in the development of resistance to fluoroquinolone (Gill et al., 1999; Jonas et al., 2001; Ng et al., 1994; Yoshida et al., 1990b).

\section{Involvement of an efflux pump in the development of resistance to fluoroquinolones}

Reserpine (a competitive pump blocker), verapamil (a calcium-channel blocker) and lansoprazole $\left(\mathrm{a} \mathrm{H}^{+}\right.$and $\mathrm{K}^{+}$ ATPase pump inhibitor) are known to inhibit various bacterial MDRs, including NorA (Aeschlimann et al., 1999) and PmrA (Gill et al., 1999). Therefore, we examined the susceptibility of the first- and second-step mutants to SPX, NOR and EtBr, some of the major substrates of NorA, with or without these inhibitors. As shown in Table 4, the susceptibility of the NOR-selected first-step mutant QR19 and the QR19-derived second-step mutant QR19-55 to NOR and EtBr increased two- to fourfold in the presence of reserpine, verapamil or lansoprazole. However, the susceptibility of these two mutants to SPX did not change. The susceptibility changes observed for QR19 and QR19-55 in the presence of MDR inhibitors were very similar to those observed for Staph. aureus RN4220 and its NorA-overexpressed derivative in the presence of the same inhibitors. It has been reported that NorA actively transports NOR and other hydrophilic fluoroquinolones out of bacterial cells, resulting in a decrease in the intracellular concentration of these drugs (Yoshida et al., 1990b). Indeed, decreased NOR accumulation was detected in the mutants QR19 and QR1955. In addition, when the protonophore CCCP was added, the intracellular concentration of NOR in QR19 and QR19-55 became almost the same as that in the wild-type strain ATCC 19434 (data not shown). These results are similar to those of previous reports that show that NorA mediates fluoroquinolone efflux in Staph. aureus ( $\mathrm{Ng}$ et al., 1994; Yoshida et al., 1990b). Furthermore, our findings strongly suggest that a NorA-like efflux pump exists in Ent. faecium and that this pump is involved in NOR resistance in the strains QR19 and QR19-55. It would be interesting to examine whether NorA-like efflux pump 


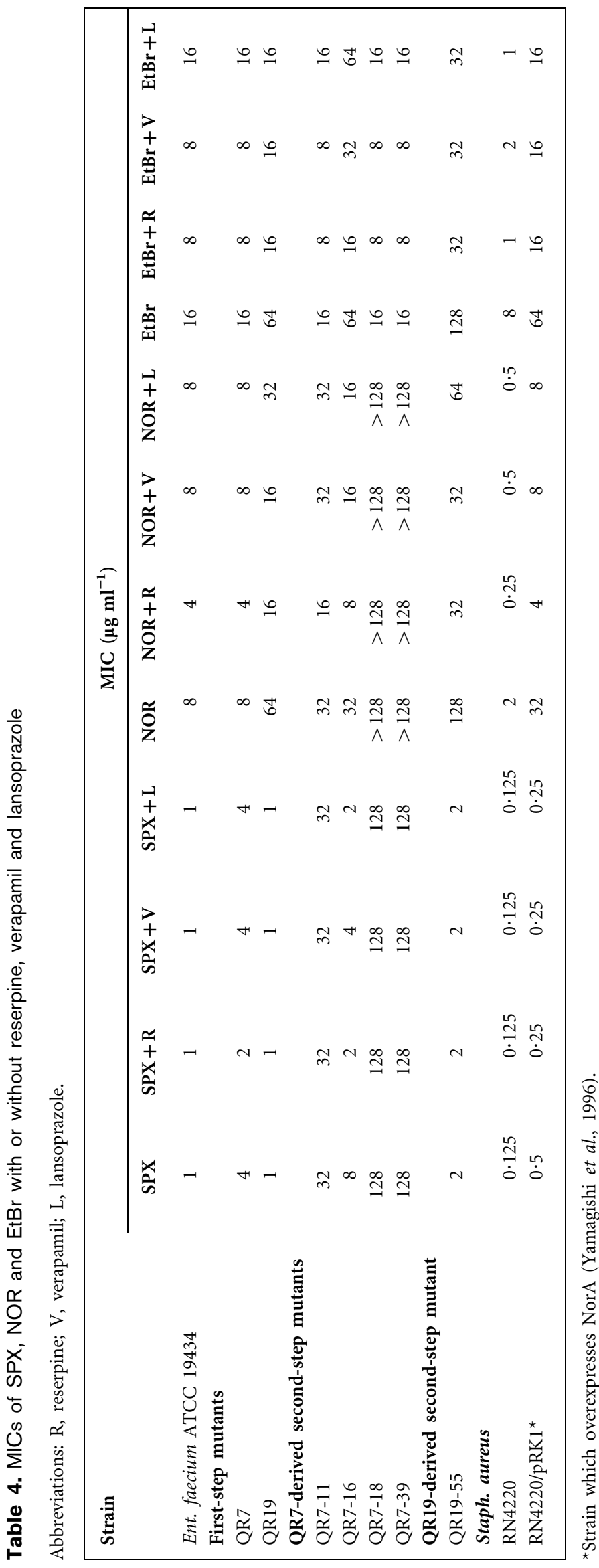

gene transcription increases, since some fluoroquinoloneresistant strains of Staph. aureus have an increased quantity of NorA that appears to result from either increased transcription of norA or increased stability of its mRNA (Kaatz \& Seo, 1995). Interestingly, the second-step mutant QR7-16 became fourfold less susceptible to SPX, NOR and $\mathrm{EtBr}$ in the presence of reserpine, and showed about threefold decreased NOR accumulation compared to QR7. The intracellular concentration of NOR in QR7-16 increased when CCCP was added. On the other hand, the SPX concentration in QR7-16 was similar to that in Ent. faecium ATCC 19434 (data not shown). The reason for the difference in intracellular concentration between NOR and SPX in QR7-16 is unclear; however, as SPX is very hydrophobic and can easily bind bacterial membranes, it is possible that the amount of membrane-bound SPX was measured instead of the amount of intracellular SPX. These findings suggest the existence of a novel multidrug transporter that pumps out not only NOR but also SPX from bacterial cells, while NorA and PmrA transport only hydrophilic fluoroquinolones such as NOR and CIP. MDR inhibitors did not influence or only marginally influenced the susceptibility of the first-step mutant QR7 and the QR7-derived second-step mutants QR7-11, QR7-18 and QR7-39 to NOR and SPX. As mentioned above, the mutant QR7-11, which had the gyrA point mutation of its parent QR7, did not have other mutations in the QRDRs of the gyrA, gyrB, parC and parE genes. In addition, the results of the accumulation assays demonstrated that the intracellular concentration of NOR in QR7-11 was almost the same as that in its parent strain QR7 (data not shown). However, QR7-11 was eight times and four times more resistant to SPX and NOR, respectively, than its parent strain QR7. These findings indicate that a mechanism(s) other than an efflux pump is involved in QR7-11 resistance to fluoroquinolones.

\section{Properties of clinical isolates of Ent. faecium}

Finally, we characterized 13 fluoroquinolone-resistant clinical isolates of Ent. faecium to examine to what extent the mechanism(s) of fluoroquinolone resistance found in the mutants isolated in vitro was similar to that in the clinical isolates. Properties of the 13 clinical isolates of Ent. faecium are summarized in Table 5. The six isolates KU2391, KU2415, KU1861, KU1869, KU2386 and KU2411 that showed low-level resistance to SPX and NOR (MICs of SPX and NOR, $\leqslant 4 \mu \mathrm{g} \mathrm{ml}^{-1}$ and $\leqslant 16 \mu \mathrm{g} \mathrm{ml}^{-1}$, respectively) did not have any amino acid substitution in the QRDRs of GyrA, GyrB, ParC and ParE. Also, the susceptibility of these isolates to SPX and NOR did not change in the presence of reserpine, verapamil or lansoprazole (data not shown). Brisse et al. (1999) have suggested that an amino acid substitution(s) in GyrB and/or ParE confers low- or intermediate-level fluoroquinolone resistance. Of course, this might be true in some low- or intermediatelevel fluoroquinolone-resistant isolates; however, our 
Table 5. Topoisomerase subunit changes in fluoroquinolone-resistant clinical isolates of Ent. faecium

\begin{tabular}{|c|c|c|c|c|c|c|}
\hline \multirow[t]{2}{*}{ Strain } & \multicolumn{2}{|c|}{$\operatorname{MIC}\left(\mu \mathrm{g} \mathrm{ml}^{-1}\right)$} & \multicolumn{4}{|c|}{ Amino acid substitution in QRDR } \\
\hline & SPX & NOR & GyrA & GyrB & ParC & ParE \\
\hline KU2415 & 1 & 8 & None & None & None & None \\
\hline KU1861 & 4 & 16 & None & None & None & None \\
\hline KU1869 & 4 & 16 & None & None & None & None \\
\hline KU1783 & 8 & $>128$ & Glu-87 (GAG) $\rightarrow$ Gly (GGG) & None & Ser-80 (AGC) $\rightarrow$ Ile (ATC) & None \\
\hline KU2400 & 16 & 128 & Glu-87 (GAG) $\rightarrow$ Lys (AAG) & None & Ser-80 (AGC) $\rightarrow$ Ile (ATC) & None \\
\hline KU2420 & 16 & 128 & Glu-87 (GAG) $\rightarrow$ Lys (AAG) & None & Ser-80 (AGC) $\rightarrow$ Ile (ATC) & None \\
\hline KU2409 & 16 & 128 & Ser-83 (AGT) $\rightarrow$ Ile $($ CGT $)$ & None & Ser-80 (AGC) $\rightarrow$ Ile (ATC) & None \\
\hline KU2377 & 64 & $>128$ & Ser-83 (AGT) $\rightarrow$ Arg (AGA) & Glu-475 (GAA) $\rightarrow$ Lys (AAA) & Ser-80 (AGC) $\rightarrow$ Ile $($ ATC $)$ & None \\
\hline
\end{tabular}

results suggest that an unknown mechanism(s) is also associated with low-level resistance to fluoroquinolones.

The isolates KU1783, KU2400, KU2420, KU2409, KU2379 and KU1778 had amino acid substitutions in both GyrA and ParC. The MICs of SPX and NOR for these isolates were 8-64 $\mu \mathrm{g} \mathrm{ml}^{-1}$ and $\geqslant 128 \mu \mathrm{g} \mathrm{ml}^{-1}$, respectively and were not influenced by efflux pump inhibitors (data not shown). Previous reports have shown that, in general, alterations in GyrA and/or ParC confer high-level fluoroquinolone resistance (Amin et al., 1999; Brisse et al., 1999). However, our analysis of the clinical isolates revealed that some combinations of alteration in GyrA and ParC (e.g. Ser-83 to Arg in GyrA and Ser-80 to Ile in ParC) confer high-level resistance to both NOR and SPX, while some other combinations (e.g. Glu-87 to Gly in GyrA and Ser-80 to Ile in ParC) are responsible for high- and intermediate-level resistance to NOR and SPX, respectively. Therefore, the SPX-resistance level seems to depend on the type and position of amino acid change in GyrA in Ent. faecium, as in Staph. aureus (Ito et al., 1994; Takenouchi et al., 1995), i.e. the amino acid change Ser-83 to Arg in GyrA confers high-level SPX resistance, while the amino acid change Glu-87 to Gly in GyrA confers low- or intermediate-level SPX resistance. On the other hand, alteration in ParC confers high-level resistance to NOR, regardless of the kind of amino acid change in GyrA. These findings suggest that alterations in DNA gyrase and topoisomerase IV are involved in SPX and NOR resistance, respectively, and strongly support the idea that the primary target of fluoroquinolones in Ent. faecium depends on the structure and/or physicochemical properties of the fluoroquinolone used.

It is worth noting that the mutants QR7-18 and QR7-39 with the amino acid changes Glu-87 to Lys in GyrA and Ser-80 to Ile in ParC, and Glu-87 to Lys in GyrA and Glu84 to Lys in ParC, respectively, showed high-level resistance to both SPX and NOR (Table 3). These findings are consistent with those that show that amino acid substitutions in GyrA and/or ParC confer high-level fluoroquinolone resistance (Brisse et al., 1999). However, in these mutants, other unknown mechanisms might be involved in SPX resistance, since, as mentioned above, the amino acid change Glu-87 to Lys in GyrA seems to confer low-level SPX resistance.

Interestingly, the strain KU2377 had an extra amino acid substitution, i.e. Glu-475 (GAA) to Lys (AAA) in GyrB, in addition to the amino acid substitutions in GyrA and ParC. This extra amino acid substitution has so far not been reported, and is equivalent to the substitution Glu-474 (GAA) to Lys (AAA) in GyrB in Strep. pneumoniae, which confers low-level resistance to SPX (Pan \& Fisher, 1998). Thus, there is a possibility that the substitution Glu-475 to Lys in GyrB is associated with low-level SPX resistance in Ent. faecium, although the MIC of SPX for KU2377 was identical to that for KU2379 or KU1778. In the case of KU2377, since the amino acid changes in GyrA and ParC confer high-level resistance to fluoroquinolones, the contribution of the amino acid change in GyrB to KU2377 resistance might be only slight.

The mechanism of acquisition of high-level resistance to fluoroquinolones in Ent. faecium, as in other Gram-positive organisms such as Staph. aureus and Strep. pneumoniae, i.e. alteration of target enzymes, is basically the same between in vitro mutants and clinical isolates. However, the efflux of fluoroquinolones, which leads to decreased intracellular accumulation, did not seem to occur in the clinical isolates examined in this study. Another unknown mechanism(s) is probably involved in fluoroquinolone resistance in the clinical isolates.

In summary, our analysis of fluoroquinolone-resistant Ent. faecium strains isolated both in vitro and clinically demonstrates that (i) the primary target for fluoroquinolones can differ depending on the structure of the compound used, (ii) 
a previously unreported amino acid substitution, Glu-475 (GAA) to Lys (AAA) in GyrB, exists, (iii) there is not only a NorA-like efflux pump but also a non-NorA-like efflux pump which transports NOR and SPX, and (iv) unknown mechanisms are involved in the development of fluoroquinolone resistance. These unknown mechanisms might be explained by the existence of (a) a novel efflux pump that excretes hydrophobic fluoroquinolones, (b) a point mutation(s) in DNA gyrase and/or topoisomerase IV outside QRDRs, and (c) a novel fluoroquinolone-resistance gene(s), such as $q n r$ (Tran \& Jacoby, 2002).

\section{ACKNOWLEDGEMENTS}

We thank Hiroaki Yoshida for his critical reading of the manuscript and useful discussion.

\section{REFERENCES}

Aeschlimann, J. R., Dresser, L. D., Kaatz, G. W. \& Rybak, M. J. (1999). Effects of NorA inhibitors on in vitro antibacterial activities and postantibiotic effects of levofloxacin, ciprofloxacin, and norfloxacin in genetically related strains of Staphylococcus aureus. Antimicrob Agents Chemother 43, 335-340.

Amin, N. E., Jalal, S. \& Wretlind, B. (1999). Alterations in GyrA and ParC associated with fluoroquinolone resistance in Enterococcus faecium. Antimicrob Agents Chemother 43, 947-949.

Brisse, S., Fluit, A. C., Wagner, U., Heisig, P., Milatovic, D., Verhoef, J., Scheuring, S., Köhrer, K. \& Schmitz, F.-J. (1999). Association of alterations in ParC and GyrA proteins with resistance of clinical isolates of Enterococcus faecium to nine different fluoroquinolones. Antimicrob Agents Chemother 43, 2513-2516.

Celesk, R. A. \& Robillard, N. J. (1989). Factors influencing the accumulation of ciprofloxacin in Pseudomonas aeruginosa. Antimicrob Agents Chemother 33, 1921-1926.

Ferrero, L., Cameron, B. \& Crouzet, J. (1995). Analysis of gyrA and grlA mutations in stepwise-selected ciprofloxacin-resistant mutations of Staphylococcus aureus. Antimicrob Agents Chemother 39, 1554-1558.

Gill, M. J., Brenwald, N. P. \& Wise, R. (1999). Identification of an efflux pump gene, $p m r A$, associated with fluoroquinolone resistance in Streptococcus pneumoniae. Antimicrob Agents Chemother 43, 187-189.

Gonzalez, M. A., Moranchel, A. H., Duran, S., Pichardo, A., Magana, J. L., Painter, B., Forrest, A. \& Drusano, G. L. (1985). Multipledose pharmacokinetics of ciprofloxacin administered intravenously to normal volunteers. Antimicrob Agents Chemother 28, 235-239.

Heisig, P. (1996). Genetic evidence for a role of parC mutations in development of high-level fluoroquinolone resistance in Escherichia coli. Antimicrob Agents Chemother 40, 879-885.

Hudson, M. C. \& Curtiss, R., III (1990). Regulation of expression of Streptococcus mutans genes important to virulence. Infect Immun 58, 464-470.

Ito, H., Yoshida, H., Bogaki-Shonai, M., Niga, T., Hattori, H. \& Nakamura, S. (1994). Quinolone resistance mutations in the DNA gyrase gyrA and gyrB genes of Staphylococcus aureus. Antimicrob Agents Chemother 38, 2014-2023.

Jonas, B. M., Murray, B. E. \& Weinstock, G. M. (2001). Characterization of emeA, a norA homolog and multidrug resistance efflux pump, in Enterococcus faecalis. Antimicrob Agents Chemother 45, 3574-3579.
Kaatz, G. W. \& Seo, S. M. (1995). Inducible NorA-mediated multidrug resistance in Staphylococcus aureus. Antimicrob Agents Chemother 39, 2650-2655.

Kanematsu, E., Deguchi, T., Yasuda, M., Kawamura, T., Nishino, Y. \& Kawada, Y. (1998). Alterations in the GyrA subunit of DNA gyrase and the ParC subunit of DNA topoisomerase IV associated with quinolone resistance in Enterococcus faecalis. Antimicrob Agents Chemother 42, 433-435.

Korten, V., Huang, W. M. \& Murray, B. E. (1994). Analysis by PCR and direct DNA sequencing of gyrA mutations associated with fluoroquinolone resistance in Enterococcus faecalis. Antimicrob Agents Chemother 38, 2091-2094.

Low, D. L., Keller, N., Barth, A. \& Jones, R. (2001). Clinical prevalence, antimicrobial susceptibility, and geographic resistance patterns of enterococci: results from the SENTRY antimicrobial surveillance program, 1997-1999. Clin Infect Dis 32 (Suppl. 2), S133-S145.

Moellering, R. C. (1992). Emergence of enterococcus as a significant pathogen. Clin Infect Dis 14, 1173-1176.

Morrissey, I. \& George, J. (1999). Activities of fluoroquinolones against Streptococcus pneumoniae type II topoisomerases purified as recombinant proteins. Antimicrob Agents Chemother 43, 2579-2585.

National Committee for Clinical Laboratory Standards (2003). Methods for dilution antimicrobial susceptibility tests for bacteria that grow aerobically, 6th edn. Approved standards M7-A6. Wayne, PA: National Committee for Clinical Laboratory Standards.

Ng, E. Y. W., Trucksis, M. \& Hooper, D. C. (1994). Quinolone resistance mediated by norA: physiologic characterization and relationship to $f l q B$, a quinolone resistance locus on the Staphylococcus aureus chromosome. Antimicrob Agents Chemother 38, 1345-1355.

Pan, X.-S. \& Fisher, L. M. (1998). DNA gyrase and topoisomerase IV are dual targets of clinafloxacin action in Streptococcus pneumoniae. Antimicrob Agents Chemother 42, 2810-2816.

Pan, X.-S., Ambler, J., Mehtar, S. \& Fisher, L. M. (1996). Involvement of topoisomerase IV and DNA gyrase as ciprofloxacin targets in Streptococcus pneumoniae. Antimicrob Agents Chemother 40, 2321-2326.

Pan, X.-S., Yague, G. \& Fisher, L. M. (2001). Quinolone resistance mutations in Streptococcus pneumoniae GyrA and ParC proteins: mechanistic insights into quinolone action from enzymatic analysis, intracellular levels, and phenotypes of wild-type and mutant proteins. Antimicrob Agents Chemother 45, 3140-3147.

Piddock, L. J. V. (1999). Mechanisms of fluoroquinolone resistance: an update 1994-1998. Drugs 58 (Suppl. 2), 11-18.

Takenouchi, T., Ishii, C., Sugawara, M., Tokue, Y. \& Ohya, S. (1995). Incidence of various gyrA mutants in 451 Staphylococcus aureus strains isolated in Japan and their susceptibilities to 10 fluoroquinolones. Antimicrob Agents Chemother 39, 1414-1418.

Tankovic, J., Mahjoubi, F., Courvalin, P., Duval, J. \& Leclercq, R. (1996). Development of fluoroquinolone resistance in Enterococcus faecalis and role of mutations in the DNA gyrase gyrA gene. Antimicrob Agents Chemother 40, 2558-2561.

Tran, J. H. \& Jacoby, G. A. (2002). Mechanism of plasmid-mediated quinolone resistance. Proc Natl Acad Sci U S A 99, 5638-5642.

Treitman, A. N., Yarnold, P. R., Warren, J. \& Noskin, G. A. (2005). Emerging incidence of Enterococcus faecium among hospital isolates (1993 to 2002). J Clin Microbiol 43, 462-463.

Yamagishi, J.-I., Kojima, T., Oyamada, Y., Fujimoto, K., Hattori, H., Nakamura, S. \& Inoue, M. (1996). Alterations in the DNA topoisomerase IV grlA gene responsible for quinolone resistance in Staphylococcus aureus. Antimicrob Agents Chemother 40, 1157-1163.

Yoshida, H., Bogaki, M., Nakamura, M. \& Nakamura, S. (1990a). Quinolone resistance-determining region in the DNA gyrase gyrA gene of Escherichia coli. Antimicrob Agents Chemother 34, 1271-1272. 
Yoshida, H., Bogaki, M., Nakamura, S., Ubukata, K. \& Konno, M. (1990b). Nucleotide sequence and characterization of the Staphylococcus aureus norA gene, which confers resistance to quinolones. J Bacteriol 172, 6942-6949.
Yoshida, H., Bogaki, M., Nakamura, M., Yamanaka, L. M. \& Nakamura, S. (1991). Quinolone resistance-determining region in the DNA gyrase gyrB gene of Escherichia coli. Antimicrob Agents Chemother 35, 1647-1650. 